\title{
Spin-current modulation and square-wave transmission through periodically stubbed electron waveguides
}

\author{
X. F. Wang, ${ }^{1}$ P. Vasilopoulos, ${ }^{1}$ and F. M. Peeters ${ }^{2}$ \\ ${ }^{1}$ Concordia University, Department of Physics, 1455 de Maisonneuve Ouest, Montréal, Québec, H3G 1M8, Canada \\ ${ }^{2}$ Departement Natuurkunde, Universiteit Antwerpen (UIA), Universiteitsplein 1, B-2610, Antwerpen, Belgium
}

(Received 26 December 2001; published 16 April 2002)

\begin{abstract}
Ballistic spin transport through waveguides, with symmetric or asymmetric double stubs attached to them periodically, is studied systematically in the presence of a weak spin-orbit coupling that makes the electrons precess. By an appropriate choice of the waveguide length and of the stub parameters injected spin-polarized electrons can be blocked completely and the transmission shows a periodic and nearly-square-type behavior, with values 1 and 0 , with wide gaps when only one mode is allowed to propagate in the waveguide. A similar behavior is possible for a certain range of the stub parameters even when two modes can propagate in the waveguide and the conductance is doubled. Such a structure is a good candidate for establishing a realistic spin transistor. A further modulation of the spin current can be achieved by inserting defects in a finite-number stub superlattice. Finite-temperature effects on the spin conductance are also considered.
\end{abstract}

DOI: 10.1103/PhysRevB.65.165217

PACS number(s): 72.20.-i, 72.30.+q, 73.20.Mf

\section{INTRODUCTION}

Spintronics-based quantum computation systems are expected to be one of the important successors of the microelectronic-based conventional computation systems in the future. The essential processes of realizing spin computation, spin injection into devices, and spin-related transport in semiconductors have attracted enthusiastic attention in the past few years. To avoid the practical difficulty of integrating devices, some electrical methods, instead of conventional ones applying an external magnetic field or employing circularly polarized light, are required to induce spin-polarized carriers in semiconductor devices. One simple idea is to use the ferromagnet-semiconductor interface to produce spinpolarized electrons, but this method must face the mismatch of physical parameters between these two quite different materials. ${ }^{1,2}$ The employment of diluted magnetic semiconductors (DMS's), which can match well with other extensively used semiconductors like $\mathrm{AlGaAs}$, has provoked a lot of interest in DMS's. ${ }^{3-6}$ Recently, based on the Rashba spinorbit interaction - an intrinsic effect in inversely asymmetric or asymmetrically confined nanostructures of nonmagnetic semiconductors (NMS's) - several designs have been proposed to spin-polarize electronic currents in nanostructures. ${ }^{7,8}$ This progress in spintronics offers the possibility of doing the spin injection in conventional materials and is bringing more and more focus on how to control and utilize the Rashba effect in these well-known materials and well-controlled structures. The possibility of establishing a spin transistor, based on the Rashba interaction, has also been considered, but further investigation is required to obtain devices of good behavior.

Spin degeneracy of carriers in semiconductors is a result of inversion symmetry, in space and time, of the considered system. By introducing a spatial inverse asymmetry, one can realize spin splitting for carriers of finite momentum, without applying any external magnetic field. This so-called Rashba spin-orbit interaction ${ }^{9,10}$ has been confirmed experimentally in different semiconductor structures. ${ }^{11-14}$ In semiconductor heterostructures, this spatial inverse asymmetry can be easily obtained by either built-in and external electric fields or by the position-dependent band edges. It is found that in many cases, especially in narrow-gap semiconductor structures, the corresponding spin-orbit interaction is a linear function of the electronic momentum $\mathbf{k}$ expressed as the Rashba term $\vec{\sigma} \cdot(\mathbf{k} \times \mathbf{E})$ in the electron Hamiltonian, where $\vec{\sigma}$ is the Pauli spin matrix and $\mathbf{E}$ the local electric field. Thus, a local electric field works on the electronic spin like a local magnetic field perpendicular to the directions of the electric field and of the electron momentum. The averaged Rashba parameter is proportional to the average electric field weighted by the electron probability and can be well controlled by a top (back) gate over (below) the device. Recently, Nitta et al. ${ }^{15,16}$ studied the dependence of the spin-orbit interaction on the surface electric field in an inverted InGaAs/InAlAs heterostructure and Grundler ${ }^{17}$ showed that the penetration of the electron wave function into the barrier layer can greatly enhance the spin-orbit interaction in InAs quantum wells because in this case the electrons suffer a stronger effective electric field due to the band-edge difference. The Rashba effect began to be considered as one of the powerful tools in making spintronic devices after the pioneer proposal of the spin-polarized field effect transistor by Datta and Das. ${ }^{18}$ Mireles and Kirczenow ${ }^{19}$ studied in detail the ballistic spinpolarized transport and the Rashba spin precession in a semiconductor waveguide using a tight-binding model but used somewhat large values for the strength of the Rashba parameter. Based on the fact that the spin orientation of electrons in nanostructures depends on the direction of their momentum, Kiselev and $\mathrm{Kim}^{7}$ proposed a T-shaped spin filter while Governale et al. ${ }^{8}$ announced recently the possibility of making a more effective spin filter with tunnel-coupled electron waveguides. Spin injection into nonmagnetic semiconductors with the help of magnetic metals ${ }^{2,20}$ or diluted magnetic semiconductors ${ }^{6}$ has also been intensively studied. However, it is not yet known how to effectively control the spin- 
polarized flux in those ballistic transport devices and waveguides, though ballistic electronic transport, disregarding spin polarization, has been studied in detail in the past several years. ${ }^{21-25}$ In a previous paper ${ }^{26}$ we showed briefly how spin-polarized transport can be produced and controlled in stubbed waveguides when only spin-up or spin-down electrons are injected and only one mode is propagating in the waveguide.

In the present paper we study in detail the possibility of a spin transistor which can control the flux strength (transmission rate) and spin orientation using periodically stubbed semiconductor waveguides in which the Rashba effect is present. In addition, we consider the case when two modes are allowed to propagate in the waveguide as well as that of "defects" in a periodic array of stubbed waveguides. We further consider the case of injected electrons polarized partially up and partially down and new stub shapes as well as the influence of finite temperatures on the conductance. The results obtained are mentioned in the abstract and are detailed as follows. In Sec. II we present the formalism and in Sec. III the numerical results for one (Sec. III A) and two propagating modes (Sec. III B) with spin-up injection. Results for finite superlattices with defects are considered in Sec. IIIC and the injection of electrons with their spins polarized in an arbitrary direction in Sec. III D. Conclusions follow in Sec. IV.

\section{FORMALISM}

For a typical two-dimensional (2D) electronic system in the $x-y$ plane in narrow-gap semiconductor nanostructures such as InGaAs/InAlAs quantum wells, the one-electron Hamiltonian including the lowest order of the spin-orbit interaction can be expressed as

$$
\begin{aligned}
H^{2 D}=\frac{\vec{p}^{2}}{2 m^{*}}+\frac{\alpha}{\hbar}(\vec{\sigma} \times \vec{p})_{z} & =-\frac{\hbar^{2}}{2 m^{*}} \vec{\nabla}^{2}+i \alpha\left(\sigma_{y} \frac{\partial}{\partial x}-\sigma_{x} \frac{\partial}{\partial y}\right) \\
& =\left[\begin{array}{cc}
-\frac{\hbar^{2}}{2 m^{*}} \vec{\nabla}^{2} & \alpha \nabla^{-} \\
-\alpha \nabla^{+} & -\frac{\hbar^{2}}{2 m^{*}} \vec{\nabla}^{2}
\end{array}\right],
\end{aligned}
$$

where $\vec{\nabla}^{2}=\partial^{2} / \partial x^{2}+\partial^{2} / \partial y^{2}$ and $\nabla^{ \pm}=\partial / \partial x \pm i \partial / \partial y$. The parameter $\alpha$ measures the strength of the spin-orbit coupling and is proportional to the interface electric field; $\vec{\sigma}$ $=\left(\sigma_{x}, \sigma_{y}, \sigma_{z}\right)$ denotes the spin Pauli matrices, and $\vec{p}$ is the momentum operator. In the presence of $\alpha$ we assume that the new wave function has the form

$$
\Psi\left(k_{x}, k_{y}\right)=e^{i k_{x} x+i k_{y} y} \sum_{\sigma} C^{\sigma}|\sigma\rangle=e^{i k_{x} x+i k_{y} y}\left(\begin{array}{l}
C^{+} \\
C^{-}
\end{array}\right),
$$
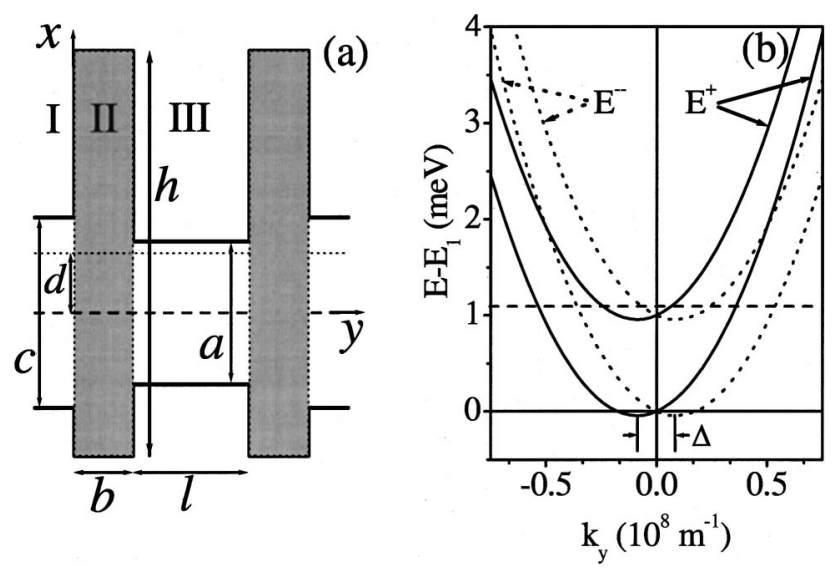

FIG. 1. (a) Schematics of a stub tuner with two units. The waveguide segment between the two stubs (shaded regions of width $b$ ) has length $l$ and widths $a$ or $c$ as indicated. The asymmetry parameter $d$ is the distance along the $y$ axis, between the lines that bisect the main waveguide and the stubs as indicated. (b) Dispersion relation for a waveguide based on Eq. (10). The horizontal dashed line indicates the four possible values of the wave vector for the same energy $E$.

with $|\sigma\rangle=\left(\begin{array}{l}1 \\ 0\end{array}\right)$ (spin up) or $\left(\begin{array}{l}0 \\ 1\end{array}\right)$ (spin down). The solutions of the equation $H^{2 D} \Psi\left(k_{x}, k_{y}\right)=E \Psi\left(k_{x}, k_{y}\right)$ are readily obtained as

$$
\Psi^{ \pm}\left(k_{x}, k_{y}\right)=\frac{1}{\sqrt{2}}\left(\begin{array}{c}
1 \\
\frac{ \pm k_{y} \mp i k_{x}}{k}
\end{array}\right) e^{i k_{x} x+i k_{y} y} ;
$$

the corresponding eigenvalues are

$$
E^{ \pm}\left(k_{x}, k_{y}\right)=\frac{\hbar^{2}}{2 m^{*}} k^{2} \pm \alpha k,
$$

where $k=\sqrt{k_{y}^{2}+k_{x}^{2}}$. The electrons are now spin polarized and oriented perpendicular to the electronic momentum in the $2 \mathrm{D}$ plane.

If the electron gas is confined along the $x$ direction by a potential $V(x)$, such as the one in the stubbed waveguide shown in Fig. 1(a), we have a quasi-one-dimensional (Q1D) electronic system. The Hamiltonian becomes

$$
H^{\mathrm{Q} 1 \mathrm{D}}=\left[\begin{array}{cc}
-\frac{\hbar^{2}}{2 m^{*}} \vec{\nabla}^{2}+V(x) & \alpha \nabla^{-} \\
-\alpha \nabla^{+} & -\frac{\hbar^{2}}{2 m^{*}} \vec{\nabla}^{2}+V(x)
\end{array}\right] .
$$

Denoting by $\phi_{n}(x)$ the solutions of the equation $\left[-\left(\hbar^{2} / 2 m^{*}\right) \partial^{2} / \partial x^{2}+V(x)\right] \phi_{n}(x)=E_{n} \phi_{n}(x)$, we can express the Q1D eigenstates in the form

$$
\Psi_{k_{y}}(x, y)=e^{i k_{y} y} \sum_{n, \sigma} \phi_{n}(x) C_{n, \sigma}|\sigma\rangle=e^{i k_{y} y} \sum_{n} \phi_{n}(x)\left(\begin{array}{c}
C_{n}^{+} \\
C_{n}^{-}
\end{array}\right) ;
$$


in each of the regions I, II, or III we have $\phi_{n}(x)=\sin [n \pi(x+w / 2) / w]$, where $w$ is the width of the region along $x$. Then the equation $H^{\mathrm{Q} 1 \mathrm{D}} \Psi=E \Psi$ takes the form

$$
\sum_{n}\left(\begin{array}{c}
{\left[E_{n}+\hbar^{2} k_{y}^{2} / 2 m^{*}-E\right] \phi_{n} C_{n}^{+}+\alpha k_{x} \phi_{n} C_{n}^{-}+\alpha \phi_{n}^{\prime} C_{n}^{-}} \\
\alpha k_{y} \phi_{n} C_{n}^{+}-\alpha \phi_{n}^{\prime} C_{n}^{+}+\left[E_{n}+\hbar^{2} k_{y}^{2} / 2 m^{*}-E\right] \phi_{n} C_{n}^{-}
\end{array}\right)=0,
$$

where $\phi_{n}^{\prime}=d \phi / d x$.

Mutiplying both sides with $\phi_{m}(x)$ and integrating over $x$ leads to $\left[\int d x \phi_{m}(x) \phi_{n}(x)=\delta_{m n}\right]$

$$
\left(\begin{array}{c}
{\left[E_{m}+\hbar^{2} k_{y}^{2} / 2 m^{*}-E\right] C_{m}^{+}+\alpha k_{x} C_{m}^{-}+\sum_{n} \int d x \alpha \phi_{m} \phi_{n}^{\prime} C_{n}^{-}} \\
\alpha k_{x} C_{m}^{+}-\sum n \int d x \alpha \phi_{m} \phi_{n}^{\prime} C_{n}^{+}+\left[E_{m}+\hbar^{2} k_{y}^{2} / 2 m^{*}-E\right] C_{m}^{-}
\end{array}\right)=0 .
$$

According to degenerate perturbation theory, if the inequality

$$
\left|\frac{\left(H_{s o}\right)_{n m}}{E_{m}-E_{n}}\right|=\left|\frac{\alpha \int d x \phi_{m} \phi_{n}^{\prime}}{E_{m}-E_{n}}\right| \ll 1
$$

holds, we can neglect the subband mixing term $\int d x \phi_{m} \phi_{n}^{\prime}$. Then Eq. (8) becomes

$$
\left[\begin{array}{cc}
E_{m}+\left(\hbar^{2} / 2 m^{*}\right) k_{y}^{2}-E & \alpha k_{y} \\
\alpha k_{y} & E_{m}+\left(\hbar^{2} / 2 m^{*}\right) k_{y}^{2}-E
\end{array}\right]\left(\begin{array}{c}
C_{m}^{+} \\
C_{m}^{-}
\end{array}\right)=0
$$

and its eigenvalues are

$$
E^{ \pm}\left(k_{y}\right)=E_{m}+\left(\hbar^{2} / 2 m^{*}\right) k_{y}^{2} \pm \alpha k_{y} .
$$

The eigenvectors corresponding to $E^{+}, E^{-}$satisfy $C_{m}^{+}=$ $\pm C_{m}^{-}$. Accordingly, the spin eigenfunctions are taken as

$$
| \pm\rangle=\frac{1}{\sqrt{2}}\left(\begin{array}{c}
1 \\
\pm 1
\end{array}\right)
$$

The dispersion relation $E^{ \pm}\left(k_{y}\right)$ versus $k_{y}$ resulting from Eq. (10) is shown in Fig. 1(b). Note that the subband minimum is shifted from $k_{y}=0$ by the value $\Delta / 2=m * \alpha / \hbar^{2}$. In each subband, electrons of the same energy $E$ have four different momentum values $k_{y}$, i.e., the positive or negative $k_{y}^{+}$and $k_{y}^{-}$values pertaining to the branches $E^{+}\left(k_{y}\right)$ and $E^{-}\left(k_{y}\right)$. In fact, electrons belonging to different branches have opposite spin orientation in the $x$ direction. The difference in wave vectors $k_{y}^{+}$and $k_{y}^{-}$, resulting from $E^{+}=E^{-}=E$, reads

$$
k_{y}^{-}-k_{y}^{+}=2 m^{*} \alpha / \hbar^{2}=\Delta .
$$

Now let us consider the transmission process when an electron of energy $E$ is incident from the left to a stubbed waveguide as illustrated in Fig. 1(a). The electron wave function is decomposed into the plus $\left(\begin{array}{l}1 \\ 1\end{array}\right)$ and minus $\left(\begin{array}{c}1 \\ -1\end{array}\right)$ branches and the procedure outlined above applies to each of the three regions labeled I, II, and III in Fig. 1(a). In each region we have $\phi_{n}(x)=\sin [n \pi(x+w / 2) / w]$, where $w$ is the width of the region along $x$. Including spin and referring to Fig. 1(b) we can write the eigenfunction of energy $E$ in region $\mathrm{I}$ as

$$
\begin{aligned}
\phi_{1}= & \sum_{m}\left\{a_{1 m}^{+} e^{i \beta_{m} y}\left(\begin{array}{l}
1 \\
1
\end{array}\right)+a_{1 m}^{-} e^{i\left(\beta_{m}+\Delta\right) y}\left(\begin{array}{c}
1 \\
-1
\end{array}\right)\right. \\
& \left.+b_{1 m}^{+} e^{-i\left(\beta_{m}+\Delta\right) y}\left(\begin{array}{l}
1 \\
1
\end{array}\right)+b_{1 m}^{-} e^{-i \beta_{m} y}\left(\begin{array}{c}
1 \\
-1
\end{array}\right)\right\} \\
& \times \sin \left[c_{m}(x+c / 2)\right]
\end{aligned}
$$

and in region III as

$$
\begin{aligned}
\phi_{2}= & \sum_{n}\left\{a_{2 n}^{+} e^{i \alpha_{n}(y-b)}\left(\begin{array}{l}
1 \\
1
\end{array}\right)+a_{2 n}^{-} e^{i\left(\alpha_{n}+\Delta\right)(y-b)}\left(\begin{array}{c}
1 \\
-1
\end{array}\right)\right. \\
& \left.+b_{2 n}^{+} e^{-i\left(\alpha_{n}+\Delta\right)(y-b)}\left(\begin{array}{l}
1 \\
1
\end{array}\right)+b_{2 n}^{-} e^{-i \alpha_{n}(y-b)}\left(\begin{array}{c}
1 \\
-1
\end{array}\right)\right\} \\
& \times \sin \left[a_{n}(x+a / 2)\right]
\end{aligned}
$$

here $c_{m}=m \pi / c, \beta_{m}=\left(2 m^{*} E-c_{m}^{2}\right)^{1 / 2}, a_{n}=n \pi / a$, and $\alpha_{n}$ $=\left(2 m^{*} E-a_{n}^{2}\right)^{1 / 2}$ where $m, n=1,2,3, \ldots$ denote the order of the transverse modes. The symbols $a_{1 m}^{\sigma}, b_{1 m}^{\sigma}, a_{2 n}^{\sigma}$, and $b_{2 n}^{\sigma}$ $(\sigma= \pm)$ represent the coefficients of different electronic modes existing in the device. Similar to the procedure of matching the electronic wave function when spin is disregarded, ${ }^{22}$ in region II we use two auxiliary sets of solutions to the wave equation, one of which matches the wire on the left and the other on the right, with each vanishing elsewhere on the boundary: $\phi_{s}=\Sigma_{k}\left(\chi_{k}^{L}+\chi_{k}^{R}\right)$. The appropriate boundary conditions are 


$$
\chi_{k}^{R}(y=0, x)=0, \quad \chi_{k}^{R}(y=b, x)=\left\{\begin{array}{cl}
0, & x<-a / 2, \\
\sin \left[a_{k}(x+a / 2)\right]\left(\begin{array}{cl}
a_{2 k}^{+}+a_{2 k}^{-}+b_{2 k}^{+}+b_{2 k}^{-} \\
a_{2 k}^{+}+a_{2 k}^{-}-b_{2 k}^{+}-b_{2 k}^{-}
\end{array}\right), & -a / 2<x<a / 2, \\
0, & x>a / 2,
\end{array}\right.
$$

$$
\chi_{k}^{L}(y=0, x)=\left\{\begin{array}{cl}
0, & x<-c / 2, \\
\sin \left[c_{k}(x+c / 2)\right]\left(\begin{array}{cl}
a_{1 k}^{+}+a_{1 k}^{-}+b_{1 k}^{+}+b_{1 k}^{-} \\
a_{1 k}^{+}+a_{1 k}^{-}-b_{1 k}^{+}-b_{1 k}^{-}
\end{array}\right), & -c / 2<x<c / 2, \\
0, & x>c / 2,
\end{array}\right.
$$

and $\chi_{k}^{L}(y=b, x)=0 . \chi_{k}^{R}$ can be expanded as

$$
\chi_{k}^{R}=\sum_{n}\left\{u_{n}^{R} e^{i \gamma_{n} y}\left(\begin{array}{l}
1 \\
1
\end{array}\right)+v_{n}^{R} e^{i\left(\gamma_{n}+\Delta\right) y}\left(\begin{array}{c}
1 \\
-1
\end{array}\right)-u_{n}^{R} e^{-i\left(\gamma_{n}+\Delta\right) y}\left(\begin{array}{l}
1 \\
1
\end{array}\right)-v_{n}^{R} e^{-i \gamma_{n} y}\left(\begin{array}{c}
1 \\
-1
\end{array}\right)\right\} \sin \left[h_{n}(x+h / 2-d)\right] .
$$

Multiplying Eq. (13) by $\sin \left[h_{m}(x+h / 2-d)\right]$, integrating over $x(-a / 2 \leqslant x \leqslant a / 2)$, and using Eq. (16), we obtain

$$
u_{m}^{R}=2\left(a_{2 k}^{+}+b_{2 k}^{+}\right) I_{k m}^{R} / K_{m}^{+}, \quad v_{m}^{R}=-2\left(a_{2 k}^{-}+b_{2 k}^{-}\right) I_{k m}^{R} / K_{m}^{-},
$$

where

$$
I_{k m}^{R}=\int_{-a / 2}^{a / 2} d x \sin \left[a_{k}(x+a / 2)\right] \sin \left[h_{m}(x+h / 2-d)\right], \quad K_{m}^{ \pm}=h\left[e^{ \pm i \gamma_{m} b}-e^{\mp i\left(\gamma_{m}+\Delta\right) b}\right],
$$

and $\gamma_{m}=\left(2 m^{*} E-h_{m}^{2}\right)^{1 / 2}$. Similarly, $\chi_{k}^{L}$ can be expanded as

$$
\chi_{k}^{L}=\sum_{n}\left\{u_{n}^{L} e^{i \gamma_{n}(y-b)}\left(\begin{array}{l}
1 \\
1
\end{array}\right)+v_{n}^{L} e^{i\left(\gamma_{n}+\Delta\right)(y-b)}\left(\begin{array}{c}
1 \\
-1
\end{array}\right)-u_{n}^{L} e^{-i\left(\gamma_{n}+\Delta\right)(y-b)}\left(\begin{array}{l}
1 \\
1
\end{array}\right)-v_{n}^{L} e^{-i \gamma_{n}(y-b)}\left(\begin{array}{c}
1 \\
-1
\end{array}\right)\right\} \sin \left[h_{n}(x+h / 2-d)\right],
$$

where

$$
u_{m}^{L}=2\left(a_{1 k}^{+}+b_{1 k}^{+}\right) I_{k m}^{L} / K_{m}^{-}, \quad v_{m}^{L}=-2\left(a_{1 k}^{-}+b_{1 k}^{-}\right) I_{k m}^{L} / K_{m}^{+}
$$

and

$$
I_{k m}^{L}=\int_{-c / 2}^{c / 2} d x \sin \left[c_{k}(x+c / 2)\right] \sin \left[h_{m}(x+h / 2-d)\right]
$$

Requiring the continuity of the derivative of the wave function at the interfaces, $\phi_{1}$ and $\phi_{2}$ must satisfy the following equation:

$$
\left[\begin{array}{cccc}
\hat{B}+\hat{\beta} & \hat{B}-\hat{\beta}^{\prime} & 0 & 0 \\
\hat{F} & \hat{F} & 0 & 0 \\
0 & 0 & \hat{D}-\hat{\beta} & \hat{D}+\hat{\beta}^{\prime} \\
0 & 0 & \hat{H} & \hat{H}
\end{array}\right]\left(\begin{array}{c}
\hat{a}_{1}^{+} \\
\hat{b}_{1}^{+} \\
\hat{a}_{1}^{-} \\
\hat{b}_{1}^{-}
\end{array}\right)=\left[\begin{array}{cccc}
\hat{A} & \hat{A} & 0 & 0 \\
\hat{E}-\hat{\alpha} & \hat{E}+\hat{\alpha}^{\prime} & 0 & 0 \\
0 & 0 & \hat{C} & \hat{C} \\
0 & 0 & \hat{G}+\hat{\alpha} & \hat{G}-\hat{\alpha}^{\prime}
\end{array}\right]\left(\begin{array}{c}
\hat{a}_{2}^{+} \\
\hat{b}_{2}^{+} \\
\hat{a}_{2}^{-} \\
\hat{b}_{2}^{-}
\end{array}\right) .
$$

The elements of submatrices $\hat{A}, \hat{B}, \hat{C}, \hat{D}, \hat{E}, \hat{F}, \hat{G}, \hat{H}, \hat{\alpha}, \hat{\beta}$, $\hat{\alpha}^{\prime}$, and $\hat{\beta}^{\prime}$ have the values

$$
B_{l k}=\sum_{m} 4\left[\gamma_{m} e^{-i \gamma_{m} b}+\left(\gamma_{m}+\Delta\right) e^{i\left(\gamma_{m}+\Delta\right) b}\right] I_{l m}^{L} I_{k m}^{L} /\left(c K_{m}^{-}\right),
$$

$$
A_{l k}=\sum_{m} 4\left(2 \gamma_{m}+\Delta\right) I_{l m}^{L} I_{k m}^{R} /\left(c K_{m}^{+}\right)
$$

$$
C_{l k}=\sum_{m}-4\left(2 \gamma_{m}+\Delta\right) I_{l m}^{L} I_{k m}^{R} /\left(c K_{m}^{-}\right)
$$




$$
\begin{gathered}
D_{l k}=\sum_{m}-4\left[\gamma_{m} e^{i \gamma_{m} b}+\left(\gamma_{m}\right.\right. \\
\left.+\Delta) e^{-i\left(\gamma_{m}+\Delta\right) b}\right] I_{l m}^{L} I_{k m}^{L} /\left(c K_{m}^{+}\right) \\
E_{l k}=\sum_{m} 4\left[\gamma_{m} e^{i \gamma_{m} b}+\left(\gamma_{m}+\Delta\right) e^{-i\left(\gamma_{m}+\Delta\right) b}\right] I_{l m}^{R} I_{k m}^{R} /\left(a K_{m}^{+}\right) \\
F_{l k}=\sum_{m} 4\left(2 \gamma_{m}+\Delta\right) I_{l m}^{R} I_{k m}^{L} /\left(a K_{m}^{-}\right) \\
G_{l k}=\sum_{m}-4\left[\gamma_{m} e^{-i \gamma_{m} b}+\left(\gamma_{m}\right.\right. \\
\left.+\Delta) e^{i\left(\gamma_{m}+\Delta\right) b}\right] I_{l m}^{R} I_{k m}^{R} /\left(a K_{m}^{-}\right)
\end{gathered}
$$

$$
H_{l k}=\sum_{m}-4\left(2 \gamma_{m}+\Delta\right) I_{l m}^{R} I_{k m}^{L} /\left(a K_{m}^{+}\right)
$$

where $\beta_{l k}=\beta_{k} \delta_{l k}, \alpha_{l k}=\alpha_{k} \delta_{l k}, \beta_{l k}^{\prime}=\left(\beta_{k}+\Delta\right) \delta_{l k}$, and $\alpha_{l k}^{\prime}$ $=\left(\alpha_{k}+\Delta\right) \delta_{l k}$.

Together with the matrix $\hat{P}$ corresponding to a waveguide segment of length $l$ rather than to a stub,

$$
\left(\begin{array}{c}
\hat{a}_{1}^{+} \\
\hat{b}_{1}^{+} \\
\hat{a}_{1}^{-} \\
\hat{b}_{1}^{-}
\end{array}\right)=\hat{P}\left(\begin{array}{c}
\hat{a}_{2}^{+} \\
\hat{b}_{2}^{+} \\
\hat{a}_{2}^{-} \\
\hat{b}_{2}^{-}
\end{array}\right)=\left[\begin{array}{cccc}
e^{-i \alpha_{m} l} & 0 & 0 & 0 \\
0 & e^{i\left(\alpha_{m}+\Delta\right) l} & 0 & 0 \\
0 & 0 & e^{-i\left(\alpha_{m}+\Delta\right) l} & 0 \\
0 & 0 & 0 & e^{i \alpha_{m} l}
\end{array}\right]\left(\begin{array}{c}
\hat{a}_{2}^{+} \\
\hat{b}_{2}^{+} \\
\hat{a}_{2}^{-} \\
\hat{b}_{2}^{-}
\end{array}\right)
$$

we can connect the incident waves (to the left of region I) with the outgoing ones (to the right of region III) via a transfer matrix $\hat{M}$ :

$$
\left(\begin{array}{c}
\hat{a}_{\text {in }}^{+} \\
\hat{b}_{\text {in }}^{+} \\
\hat{a}_{\text {in }}^{-} \\
\hat{b}_{\text {in }}^{-}
\end{array}\right)=\hat{M}\left(\begin{array}{c}
\hat{a}_{\text {out }}^{+} \\
\hat{b}_{\text {out }}^{+} \\
\hat{a}_{\text {out }}^{-} \\
\hat{b}_{\text {out }}^{-}
\end{array}\right) .
$$

Here $\hat{M}$ is a $4 \times 4$ matrix with submatrices $\hat{M}_{m n}$ as its elements. If we assume that a spin-coherent electron beam of single energy is injected into the device from the left and it is detected at its right end by an analyzer, there is no backward propagating wave at the output and $\hat{b}_{\text {out }}^{+}=\hat{b}_{\text {out }}^{-}=0$. The coefficients of spin-up and spin-down electrons are $\hat{a}_{i n}^{+}+\hat{a}_{i n}^{-}$ and $\hat{a}_{\text {in }}^{+}-\hat{a}_{\text {in }}^{-}$for the input wave and $\hat{a}_{\text {out }}^{+}+\hat{a}_{\text {out }}^{-}$and $\hat{a}_{\text {out }}^{+}$ $-\hat{a}_{\text {out }}^{-}$for the output wave, respectively, if we neglect the injection mismatch of electronic momentum between different electron branches. Then the spin-dependent transmission rate can be calculated similar to the procedure used when the spins are disregarded.

In the design of a spin transistor first proposed by Datta and Das, we can introduce the above stubbed waveguide together with the Rashba effect into the device to control and flip the spin current. To see this effect clearly, we connect a spin polarizer (analyzer) to the left (right) of the structure and inject spin-up polarized electrons into it and detect the polarization of the outgoing electrons. The electron beam is equally decomposed into the plus branch $\left(\begin{array}{l}1 \\ 1\end{array}\right)$ and the minus branch $\left(\begin{array}{c}1 \\ -1\end{array}\right)$ if the momentum difference between two branches can be neglected when considering the wave function match of input and output. Since electrons belonging to different branches will get different phases when propagating along the device, the output spin orientation of the electrons, which is a result of the composition of the electronic wave function of the two branches when leaving from the waveguide, can be different from the initial one. In our case, the spin-up transmission rate $T^{+}$and spin-down transmission rate $T^{-}$read

$$
T^{ \pm}=\sum_{m n} T_{m n}^{ \pm}=\sum_{m n} \frac{\alpha_{n}\left|a_{\text {out }, m n}^{+} \pm a_{\text {out }, m n}^{-}\right|^{2}}{2 \beta_{m}\left(\left|a_{\text {in, } m}^{+}\right|^{2}+\left|a_{\text {in }, m}^{-}\right|^{2}\right)},
$$

where $a_{\text {out }, m n}$ denotes the $m$ th mode input contribution to the $n$th mode output $a_{\text {out }, n}$ and the sums over $m, n$ are over all possible propagating modes (number $N_{m}$ ) in the waveguide. For spin-up electrons injected into a simple waveguide of length $l$, we can assume $a_{i n, m}^{+}=a_{i n, m}^{-}=1 / 2$ and the output coefficients can be calculated using Eq. (31): $a_{\text {out }, m n}^{+}$ $=e^{-i \alpha_{m} l} \delta_{m n}$ and $a_{\text {out }, m n}^{-}=e^{-i\left(\alpha_{m}+\Delta\right) l} \delta_{m n}$. Since $\alpha_{n}=\beta_{n}$ for a simple waveguide, we have $T^{ \pm}=N_{m}\left|1 \pm e^{-i \Delta l}\right|^{2}$. The probability of detecting a spin-down $\left(\begin{array}{l}0 \\ 1\end{array}\right)$ electron will be $T^{-}=N_{m} \sin ^{2}(\delta \theta / 2)($ Refs. 18 and 19) and that for a spin-up $\left(\begin{array}{l}1 \\ 0\end{array}\right)$ electron $T^{+}=N_{m} \cos ^{2}(\delta \theta / 2)$, where $\delta \theta=\Delta l$ is the phase difference between the two spin propagation modes $|+\rangle$ and $|-\rangle$ in the structure after traveling a distance $l$. In stubbed 
waveguides, it will be shown that the percentage of transmitted spin-up and spin-down electrons follows that same rule as in a stubless waveguide when the Rashba effect is weak. The advantage of stubbed waveguides is that using side gates to control the length $h$ of the stubs and the distance $d$ of their centers from that of the waveguide, we can control the transmission rate. At the same time we can adjust the back gate bias to change the Rashba parameter and then the outgoing spin orientation.

Once $T^{ \pm}$is known, the conductance $G^{ \pm} \equiv G(E, 0)$ at zero temperature is given by the Landauer-Büttiker formula $G^{ \pm}$ $=\left(2 e^{2} / h\right) T^{ \pm}$. For finite temperatures the conductance $G(E, T)$ is given by

$$
G(E, T)=\int_{-\infty}^{\infty} G(\epsilon, T=0)\left(-\frac{d f}{d \epsilon}\right) d \epsilon
$$

where $f(\epsilon-E)$ is the Fermi function.

In real devices the shape of the stubs can be different than a rectangular one and one question we need to answer is the influence of the shape of the stubs on the transmission output. It is known from electronic stub tuners that stubs of different shape, e.g., Lorentzian or triangular stubs, do not change the qualitative behavior of the transmission. To quantitatively study the transmission rate of electronic current through a waveguide with arbitrarily shaped stub, we can break the stubs into a series of rectangular segments with the same width $b_{i}$ and different heights. Each segment is described by a transfer matrix $M_{i}$. The complete shape is well described by the product $M^{T}=\Pi_{i} M_{i}$ if the segment width is much smaller than the electronic wavelength. The entire procedure described above for a single stub is then repeated each segment as many times as required by the particular shape.

\section{RESULTS AND DISCUSSION}

In the numerical calculations we consider a stubbed $\mathrm{In}_{0.53} \mathrm{Ga}_{0.47} \mathrm{As} / \mathrm{InAlAs}$ waveguide. The effective electron mass is $m^{*}=0.042 m_{0}$ and a typical average spin-orbit constant $\alpha=1.6 \times 10^{-11} \mathrm{eVm}$ is assumed throughout the paper unless otherwise specified. To verify the validity of the perturbation theory we evaluated the bound states of one unit, of stub length $h=2000 \AA$ and width $b=150 \AA$, connected to a waveguide of width $a=250 \AA$ and segment length $l$ $=100 \AA$ to the left and right of the stub. We find that the ratio of the intersubband mixing energy over the difference between the lowest two bound states is less than $10 \%$. We also calculated the energy bands of a superlattice of such stub units with a waveguide segment of length $l=200 \AA$ between two consecutive stubs. The separation of the two spin bands is less than $15 \%$ of the band energy.

\section{A. One mode allowed in the waveguide}

\section{Rectangular stubs}

To view the parameter dependence of the spin transmission through a stubbed waveguide, we inject spin-up polarized electrons into a waveguide of width $a=250 \AA$ with a

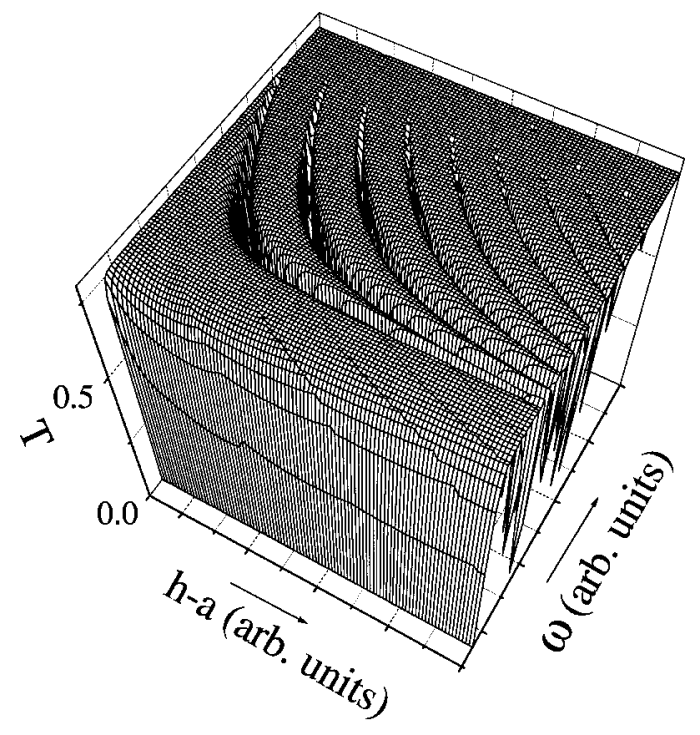

FIG. 2. Three-dimensional illustration of transmission $T$ vs electron energy and stub arm length $h-a$ of a symmetric stub tuner.

symmetric double stub of width $b=150 \AA$ and measure the output flux rate of spin-up and spin-down electrons. Considering only the first mode and for a waveguide segment of length $l=450 \AA$, we plot in Fig. 2 the spin-up electron transmission as a function of the stub length, reduced by the width of the waveguide $(h-a)$, and of the electron energy $E$. Only electrons with energy higher than the first subband in the waveguide, $E_{1}=\pi^{2} /\left(2 m^{*} a^{2}\right)=14.3 \mathrm{meV}$ can pass through the device, and the output percentage of spin-up and spin-down electrons depends only on the total length of the device. Here $\Delta=2 m^{*} \alpha / \hbar^{2}=0.1764 \times 10^{8} \mathrm{~m}^{-1}$, so the maximum of the spin-up electron transmission rate is $\cos ^{2} \Delta(b+l)=76.4 \%$ and the maximum of the spin-down electron transmission rate $23.6 \%$. The stub begins to play a role in adjusting the transmission rate when electrons have an energy higher than the first subband in the stub, $E_{1}^{s}$ $=\pi^{2} /\left(2 m^{*} b^{2}\right)=57.2 \mathrm{meV}$. If the electronic energy has a value between the first and second transverse subbands, i.e., if the relation $E_{1}^{s}<E<E_{2}^{s}=2 \pi^{2} /\left(m^{*} b^{2}\right)$ holds, only one transverse mode enters the stub and a simple transmission pattern appears with transmission gaps along the curves $h$ $=n \lambda+h_{0}$, where $h_{0}$ is the position of the first gap and $n$ $=0,1,2, \ldots$. Notice that the period $\lambda$ is different from the $x$-direction wavelength of the first subband wave function in the stub, $\lambda_{1}=2 \pi b / \sqrt{2 m^{*} E b^{2}-\pi^{2}}$, and depends on the parameters $a$ and $l$. When the width $b$ of the stub becomes wider, more modes can exist in the stub and the transmission pattern becomes complicated; see Sec. III C.

We can also change the asymmetry parameter $d$ - the distance along $x$ between the midpoint of the waveguide and that of the height $h$ shown in Fig. 1(a) - to shift the stub along the $x$ direction so the structure becomes asymmetric. In Fig. 3 we show by a wired surface the transmission rate for electrons of fixed energy $E=48 \mathrm{meV}$ as a function of $d$ and $h$ for a five-stub device with parameters $a=c=250 \AA, b$ $=150 \AA$, and $l=207.5 \AA$. The parameter $l$ is chosen to satisfy $\cos ^{2}[5 \Delta(b+l)]=0$ so that spin-up electrons are totally 


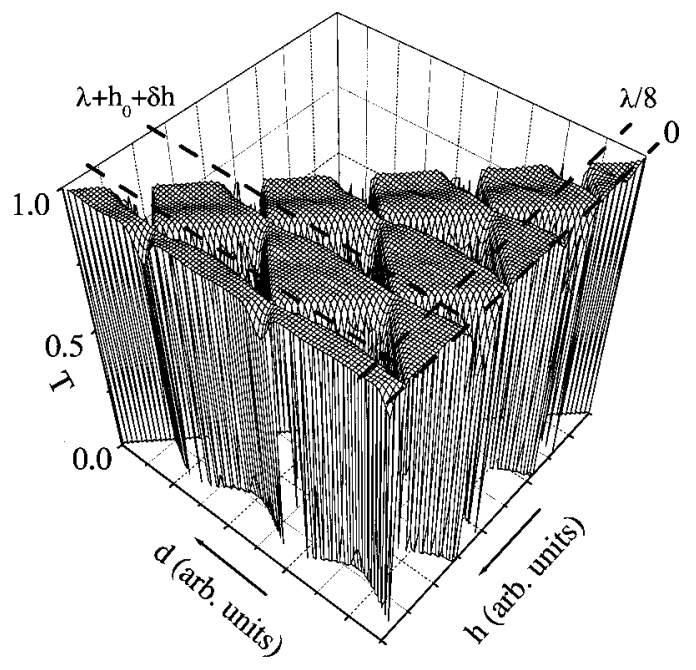

FIG. 3. Spin-down transmission of an array of five stub tuners as a function of the stub length $h$ and stub shift $d$ when only spin-up electrons are injected. Cross sections along the dashed lines are shown in Fig. 4.

blocked $\left(T^{+}=0\right)$, and only spin-down electrons come out. The transmission is a periodic function of $d$ and $h$, and the gaps appear in the triangle-shaped regions. The centers of these triangles are located at the points in the $h-d$ plane that satisfy $h=n \lambda / 2+h_{0}$ and $2 d+h=n \lambda+h_{0}$ for integer $n$ $=0,1,2, \ldots$. In this figure we find $\lambda=558 \AA, h_{0}$ $=694 \AA$, and $\lambda_{1}=660.5 \AA$. One of the interesting facts found in Fig. 3 is that a square-type transmission curve with a wide gap can be obtained by changing $d$ for a fixed value of $h$.

To clearly show when and how these square-shaped transmission curves appear, some cross sections of Fig. 3 are shown in Fig. 4. In Fig. 4(a) the spin-down transmission rate is shown as a function of $h$ for the symmetric case $(d=0)$. Gaps appear with period $\lambda$, and the first gap begins at $h$ $=h_{0}=694 \AA$ and has a width $\delta h=40 \AA$. If we cut the
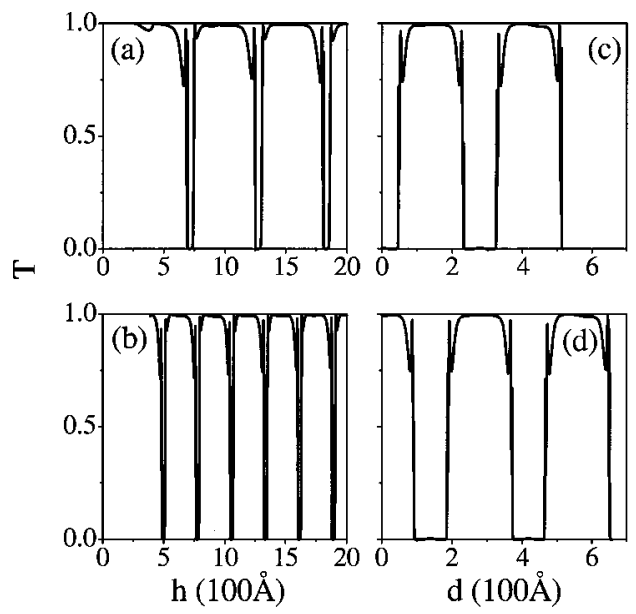

FIG. 4. Two-dimensional cross section of Fig. 3 in the transmission $T$ vs stub length $h$ for fixed $d[d=0$ in (a) and $d=\lambda / 8$ in (b)] and vs stub shift $d$ for fixed $h[h=1289.5 \AA$ in (c) and $h$ $=1568.5 \AA$ in $(\mathrm{d})]$.
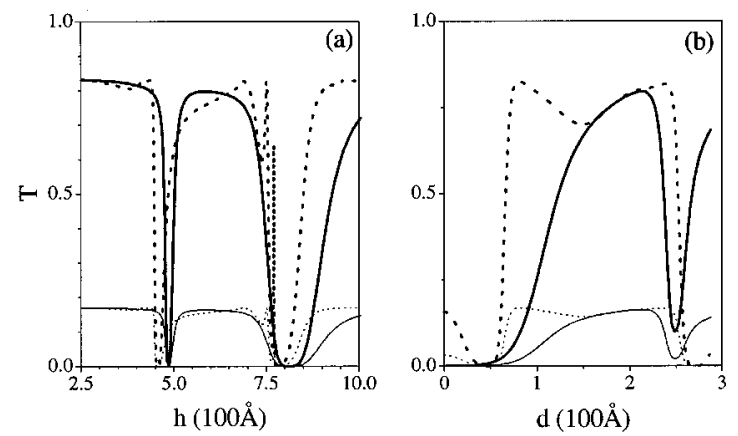

FIG. 5. Transmission as a function of the stub length $h$ for symmetric double stubs (a) and of the stub shift $d$ for asymmetric double stubs (b). The thin and thick solid curves show, respectively, the spin-up and spin-down transmission for Gaussian-shaped stubs. The thin and thick dotted curves show the corresponding results for rectangular stubs with parameters $a=250 \AA, \quad b=290 \AA$, and $L=360 \AA$.

surface along $h$ at $d=69.75 \AA=\lambda / 8$, we obtain Fig. 4(b). Here we see a periodic structure similar to that in (a) but with half the period $\lambda / 2$ and half the gap width $\delta h=20 \AA$. If we fix the value of the stub length $h$ in one of the gaps in Fig. 4(a) and cut the surface of Fig. 3 along $d$, we obtain the curve shown in (c), where $h=1289.5 \AA \simeq h_{0}+\lambda$. The transmission has a period $\lambda / 2$ along $d$ with gaps as wide as $\delta d$ $=91 \AA$. The same periodic transmission versus $d$ curves is shown (d) when we make the same cut as above but fix $h$ $=1568.5 \AA \simeq h_{0}+3 \lambda / 2$, so the gaps appear at $d=\lambda / 4$ rather than at $d=0$. We see that in (c) and (d) we can completely flip or block the input electronic spin flux and the transmission has an almost square-wave dependence on the adjustable parameter $d$ with a gap/band ratio as big as 0.5 .

\section{Stubs of general shape}

To study the influence of the stub shape on the transmission output we proceed as outlined at the end of Sec. II. As an example we consider devices with double stubs of Gaussian shape. As a function of $x$ the $y$ coordinate of the boundary of each stub is taken as $y=[(h-a) / 2 \pm d]\left(e^{-8 x^{2} / b^{2}}\right.$ $\left.-e^{-2}\right) /\left(1-e^{-2}\right)$. In Fig. 5(a) we show the transmission of a structure of two symmetric double stubs as a function of the total length $h$ of the stubs. Only spin-up electrons are injected. The thin and thick solid curves show, respectively, the spin-up and spin-down transmission for Gaussian-shaped stubs and parameters $a=250 \AA, b=375 \AA$, and $l$ $=275 \AA$; the thin and thick dotted curves are for rectangular shapes with parameters $a=250 \AA, b=290 \AA$, and $L$ $=360 \AA$. We see that the transmission through the waveguide with Guassian-shaped stubs is similar to that with narrower rectangular stubs for the same total length $(b+L)$. Such a similarity was noticed earlier between rectangular and triangular stubs. ${ }^{26}$ If we fix the total length of the stubs of both waveguides, $h=825 \AA$, and shift the stubs, we obtain Fig. 5(b) for the transmission as a function of the shift $d$.

\section{B. Two modes allowed in the waveguide}

When the electron energy is high enough, two or more transverse modes can propagate in the waveguide. In Fig. 

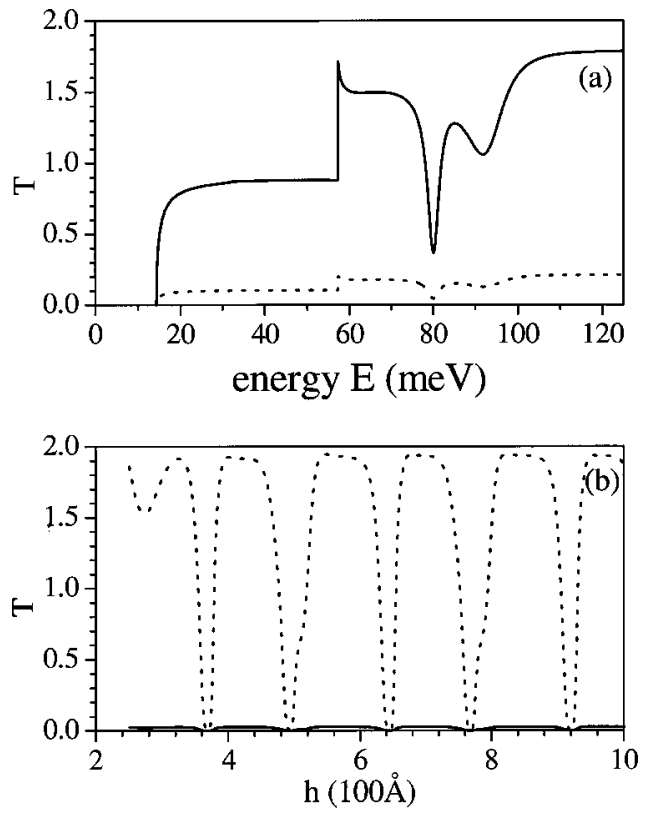

FIG. 6. (a) Spin-up $T^{+}$(solid line) and spin-down $T^{-}$(dotted line) transmission through one stub tuner as a function of the electron energy $E$. (b) $T^{+}$and $T^{-}$for four identical stub tuners as a function of the stub length $h$.

6(a), we plot the transmission as a function of the electronic energy $E$ through a device with one waveguide segment of width $a=250 \AA$ and length $l=250 \AA$ and one stub of width $b=125 \AA$ and length $h=1250 \AA$. Here the total device length is adjusted so that both spin-up (solid line) and spin-down (dotted line) electrons can be observed at the right end. When the electron energy is lower than $E_{2}$ $=57.2 \mathrm{meV}$, only one propagating mode exists and the transmission is almost flat; the total transmission $\left(T^{+}\right.$ $+T^{-}$) approaches unity because in this energy region the stub is too narrow to give significant contribution. With the increase of the electron energy, two modes can exist in the structure. In some cases, two modes compete and a deep minimum exists with the transmission dropping from 2 to a value much lower than 1 as shown in Fig. 6(a). If a wider stub is used here, more modes can exist in the stub and the curve has many more oscillations.

In Fig. 6(b) we inject electrons of energy $100 \mathrm{meV}$ into a device of four equal units, each of which has one asymmetric stub with $b=125 \AA$ and $d=30 \AA$ connected to one waveguide segment of width $a=250 \AA$ and length $l=300 \AA$. The transmission rate is shown as a function of the length $h$ of the stubs. In this figure the spin-orbit parameter is adjusted to $\alpha=1.8 \times 10^{-11} \mathrm{eV} \mathrm{m}$ so that almost all electrons are spin flipped after passing the device. As in Fig. 4 we observe a series of gaps, narrower than the corresponding ones of Fig. 4 , but now with two modes present and electrons of either spin completely blocked. Notice that the transmission rate is still a periodic function of the stub length $h$ despite the presence of two modes.

\section{Introducing defects}

Using a series of periodically arranged stub units, we can make a spintronic crystal (superlattice) with a transmission

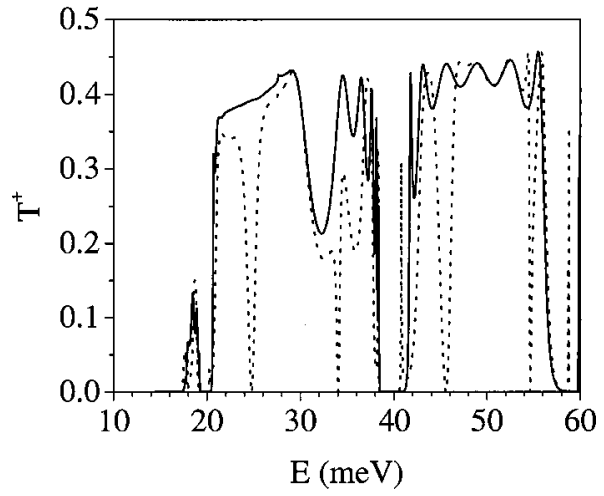

FIG. 7. Spin-up transmission $T^{+}$vs electron energy $E$ for a periodic array of seven units (solid line) and for the same structure with the central (fourth) unit replaced by a defect unit.

similar to that shown in Fig. 3, where five units are used. In this kind of crystal, we can expect to obtain devices of better behavior than that of only one unit. If we add some impurities into the crystal, we can change the properties of this crystal and observe a different transmission rate. The solid line in Fig. 7 shows the first mode spin-up transmission rate versus electron energy through a pure finite crystal of seven equal units with symmetric stubs and parameters $a$ $=250 \AA, b=250 \AA, l=125 \AA$, and $h=2250 \AA$. The transmission shows gaps near $E=20 \mathrm{meV}, 40 \mathrm{meV}$, and $60 \mathrm{meV}$. When we substitute the middle (fourth) unit by a defect-that is, a unit which has stub length $h_{d}=4500 \AA$ and otherwise the same parameters-we find that the defect introduces resonant peaks in the gaps at $E=40 \mathrm{meV}$ and 60 $\mathrm{meV}$, and resonant dips at $E=25 \mathrm{meV}, 34 \mathrm{meV}, 46 \mathrm{meV}$, and $55 \mathrm{meV}$. The spin-down transmission rate has the same structure but different scale. A similar behavior was reported for photonic tuners in Ref. 25.

\section{Injection of spin-up and spin-down electrons}

Up to now we considered electronic conductance or transmission through stubbed waveguides when only spin-up electrons are injected at zero temperature $(G(E, T=0))$. In this subsection we discuss what happens if arbitrarily spinpolarized electrons are injected into a stubbed waveguide at finite temperature. In the $\sigma_{z}$ spin representation the spin orientation is denoted by the coefficients $C^{+}$and $C^{-}$. For instance, $\left(\begin{array}{c}1 \\ \pm 1\end{array}\right)$ describes a spin oriented along the $x$-direction whereas $\left(\begin{array}{c}1 \\ \pm i\end{array}\right)$ describes a spin oriented along the $y$-direction. In Fig. 8(a) and (b) we show the spin-up (dotted line) and spin-down (solid line) conductance for a structure composed of eight stub units with $a=150 \AA, b=80 \AA, l$ $=69 \AA$ and Fermi energy $E_{F}=133 \mathrm{meV}$. The spin orientation of the incident electrons is as follows: we let $C^{+}=0.7$ and $C^{-}=-0.3$; since the state must be normalized $\left(\left|C^{+}\right|^{2}\right.$ $\left.+\left|C^{-}\right|^{2}=1\right)$, this corresponds to $84.5 \%$ spin-up and $15.5 \%$ spin-down incident electrons.

As a function of the stub length $h$ the conductances $G^{+}$ and $G^{-}$, at $T=0$ and $8 \mathrm{~K}$, are shown in Fig. 8(a); the solid and dotted curves are for $G^{+}$and the thin and thick solid curves for $G^{-}$. As a function of the shift $d G^{+}$and $G^{-}$are 

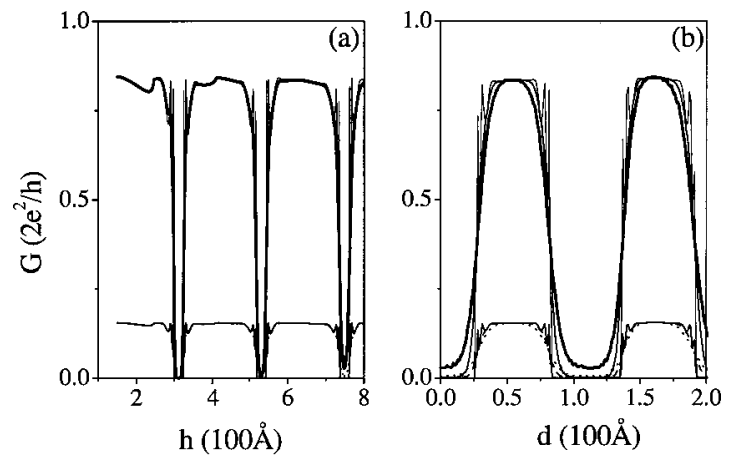

FIG. 8. Spin-up $G^{+}$(lower lines) and spin-down conductance $G^{-}$(upper lines) as functions of the stub length $h$ (a) and of the stub shift $d$ (b). The various curves and temperatures are specified in the text.

shown in Fig. 8(b) at $T=0,4.2$, and $8 \mathrm{~K}$. The solid, dashed, and dotted curves are for $G^{+}$and the thin, medium, and thick solid lines for $G^{-}$. Here we have $15.5 \%$ of the output electrons having spin up and $84.5 \%$ of them spin down. The conductance $G^{-}$shows a behavior similar to that of the transmission in Figs. 4(a) and 4(d). As can be seen, finite temperatures smoothen the curves obtained at zero temperature similar to the case of spinless electrons. ${ }^{22}$ As the ratio $E_{F} / k_{B} T$ increases the curves are smoothened or rounded off more strongly.

\section{CONCLUDING REMARKS}

Employing the transfer-matrix method we combined the spin precession in a waveguide, due to the spin-orbit coupling, with the basic physics of a stub tuner and applied it to the ballistic spin transmission through periodically stubbed waveguides. We found that the spin polarization can be well controlled by adjusting the total length of the device for a wide range of the electronic energy. In particular, we showed that, given spin-polarized electrons injected into a stub structure, we can select the spin of the outgoing electrons to be the same as or opposite to that of the injected electrons. We demonstrated this for stubs of rectangular or Gaussian shape but also for triangular stubs. ${ }^{26}$ In general these results hold for stubs of any shape. The shape does not affect the qualitative behavior of the transmission but only its period when several stubs are combined.

More important, we saw clearly that, as a function of the stub height $h$ and the asymmetry parameter $d$, we can have a nearly binary square-wave transmission (spin-valve effect) for either spin orientation, with wide gaps for stubs of different shapes and a well-controlled range of the stub parameters. In this respect asymmetric stubs give the best results. Their asymmetry, i.e., $h$ and $d$, can be controlled by lateral gates ${ }^{21}$ in principle, such gates should allow for a more detailed control of the overall stub shape. A further modulation can be achieved if we combine several groups of stubs with different values of the spin-orbit coupling strength. These findings should facilitate the experimental realization of the spin transistor.

A qualitative understanding of all these results is as follows. The spins precess in a single waveguide ${ }^{18}$ due to the spin-orbit coupling. On the other hand, in a stub tuner waves reflected from the walls of the stub, where the wave function vanishes, may interfere constructively or destructively with those propagating in the main waveguide and result, respectively, in an increase or decrease of the transmission. ${ }^{24} \mathrm{Re}$ fining this idea, it was shown in Refs. 21 and 22 that using double stubs the transmission of spinless electrons could be blocked completely using asymmetric stubs. Combining several stubs would result in a nearly square-wave transmission output, especially as a function of the asymmetry parameter $d$. The transmission shown, e.g., in Figs. 2-4 is simply the result of this behavior when combined with the spin precession due to the spin-orbit coupling since the length of the device was chosen such that spin flip would occur in the stubless waveguide.

The most clear results or simplest transmission patterns are obtained when only one mode is allowed to propagate in the main waveguide. If more modes can propagate in the main waveguide, generally the transmission pattern becomes more complex or even irregular. However, as we demonstrated, we can have a simple periodic transmission pattern even when two modes are allowed. This occurs when the stub width is short enough that only one mode enters in the stub region.

Further, we showed that the above results hold when the injected spins are polarized in an arbitrary direction, partially "up" and partially "down." We also showed that introducing "defects" in a finite superlattice leads to a further modulation of the spin current since the defects produce new transmission resonances or antiresonances. One could use such defects to achieve a specific control of the transmission.

Finally, we have seen that the effect of finite temperatures is to smoothen the zero-temperature conductance as in the case of spinless electrons. ${ }^{22}$ The degree of smoothness depends mainly on the ratio $E_{F} / k_{B} T$.

\section{ACKNOWLEDGMENTS}

The work of X.F.W. and P.V. was supported by the Canadian NSERC Grant No. OGP0121756 and that of F.M.P. by the Flemish Science Foundation, the Belgian Interuniversity Attraction Poles (IUAP), the Interuniversity Microelectronics Center (IMEC), and the Concerted action program (GOA).
${ }^{1}$ L.W. Molenkamp, G. Schmidt, and G.E.W. Bauer, Phys. Rev. B 64, R121202 (2001); E.I. Rashba, ibid. 62, R16 267 (2000); G. Schmidt, D. Ferrand, L.W. Molenkamp, A.T. Filip, and B.J. van Wees, ibid. 62, R4790 (2000).

${ }^{2}$ H.J. Zhu, M. Ramsteiner, H. Kostial, M. Wassermeier, H.P.
Schonherr, and K.H. Ploog, Phys. Rev. Lett. 87, 016601 (2001); D. Grundler, ibid. 86, 1058 (2001); P.R. Hammar, B.R. Bennett, M.J. Yang, and Mark Johnson, ibid. 83, 203 (1999); S. Gardelis, C.G. Smith, C.H.W. Barnes, E.H. Linfield, and D.A. Ritchie, Phys. Rev. B 60, 7764 (1999); H.X. Tang, F.G. Monzon, R. 
Lifshitz, M.C. Cross, and M.L. Roukes, ibid. 61, 4437 (2000).

${ }^{3}$ Y. Ohno, D.K. Young, B. Beschoten, F. Matsukura, H. Ohno, and D.D. Awschalom, Nature (London) 402, 790 (1999); R. Fiederling, M. Keim, G. Reuscher, W. Ossau, G. Schmidt, A. Waag, and L.W. Molenkamp, ibid. 402, 787 (1999); R. Fitzgerald, Phys. Today 53(4), 21 (2000); J.M. Kikkawa and D.D. Awschalom, Phys. Rev. Lett. 80, 4313 (1998).

${ }^{4}$ L. Loureiro da Silva, M.A. Boselli, X.F. Wang, and I.C. da Cunha Lima, Appl. Phys. Lett. 79, 3305 (2001); X.F. Wang, L. Loureiro da Silva, M.A. Boselli, and I.C. da Cunha Lima, Physica E (to be published); M.A. Boselli, A. Ghazali, and I.C. da Cunha Lima, Phys. Rev. B 62, 8895 (2000).

${ }^{5}$ K. Chang and F.M. Peeters, Solid State Commun. 120, 181 (2001); K. Chang, J.B. Xia, and F.M. Peeters, Phys. Rev. B 65, 115209 (2002); G. Papp and F.M. Peeters, Appl. Phys. Lett. 78, 2184 (2001); 79, 3198 (2001).

${ }^{6}$ R.N. Gurzhi, A.N. Kalinenko, A.I. Kopeliovich, A.V. Yanovsky, and B. Verkin, cond-mat/0109041 (unpublished).

${ }^{7}$ A.A. Kiseley and K.W. Kim, Appl. Phys. Lett. 78, 775 (2001).

${ }^{8}$ M. Governale, D. Boese, U. Zulicke, and C. Schroll (unpublished).

${ }^{9}$ E.I. Rashba, Fiz. Tverd. Tela (Leningrad) 2, 1224 (1960) [Sov. Phys. Solid State 2, 1109 (1960)]; Y.A. Bychkov and E.I. Rashba, J. Phys. C 17, 6039 (1984).

${ }^{10}$ R. Winkler and U. Rössler, Phys. Rev. B 48, 8918 (1993).

${ }^{11}$ H.L. Stormer and Z. Schlesinger, Phys. Rev. Lett. 51, 126 (1983).
${ }^{12}$ D. Stein and K.v. Klitzing, Phys. Rev. Lett. 51, 130 (1983).

${ }^{13}$ B. Das, D.C. Miller, and S. Datta, Phys. Rev. B 39, 1411 (1989).

${ }^{14}$ J. Luo, H. Munekata, F.F. Fang, and P.J. Stiles, Phys. Rev. B 38, 10142 (1988); 41, 7685 (1990).

${ }^{15}$ J. Nitta, T. Akazaki, H. Takayanagi, and T. Enoki, Phys. Rev. Lett. 78, 1335 (1997); C.M. Hu, J. Nitta, T. Akazaki, H. Takayanagi, J. Osaka, P. Pfeffer, and W. Zawadzki, Phys. Rev. B 60, 7736 (1999).

${ }^{16}$ G. Engels, J. Lange, Th. Schapers, and H. Luth, Phys. Rev. B 55, R1958 (1997).

${ }^{17}$ D. Grundler, Phys. Rev. Lett. 84, 6074 (2000).

${ }^{18}$ S. Datta and B. Das, Appl. Phys. Lett. 56, 665 (1990).

${ }^{19}$ F. Mireles and G. Kirczenow, Phys. Rev. B 64, 024426 (2001).

${ }^{20}$ G. Kirczenow, Phys. Rev. B 63, 054422 (2001).

${ }^{21}$ P. Debray, R. Akis, P. Vasilopoulos, and J. Blanchet, Appl. Phys. Lett. 66, 3137 (1995).

${ }^{22}$ R. Akis, P. Vasilopoulos, and P. Debray, Phys. Rev. B 52, 2805 (1995); 56, 9594 (1997); H. Wu, D.W.L. Sprung, J. Martorell, and S. Klarsfeld, ibid. 44, 6351 (1991).

${ }^{23}$ F. M. Peeters, in Science and Engineering of One-and Zerodimensional Semiconductors, edited by S.P. Beaumont and C.M. Sotomayor-Tores (Plenum Press, New York, 1990), p. 107.

${ }^{24}$ F. Sols, M. Macucci, U. Ravaioli, and K. Hess, Appl. Phys. Lett. 54, 350 (1989); S. Datta, Superlattices Microstruct. 6, 83 (1989).

${ }^{25}$ R. Akis and P. Vasilopoulos, Phys. Rev. E 53, 5369 (1996).

${ }^{26}$ X.F. Wang, P. Vasilopoulos, and F.M. Peeters (unpublished). 